\title{
Efficient Color Cast Correction Based on Fuzzy Logic
}

\author{
Om Prakash Verma ${ }^{1}$ and Nitin Sharma ${ }^{2, *}$ \\ ${ }^{1}$ Department of Computer Science Engineering, Delhi Technological University, Delhi, India \\ ${ }^{2}$ Department of Electronic and Communication Engineering, Maharaja Agrasen Institute of Technology, Rohini, Delhi, India
}

Received 31 October 2016; Accepted 6 July 2017

\begin{abstract}
Color cast defect detection of an image is challenging and of great importance in the field of image quality evaluation. This paper presents a simple and less complex method to improve the visibility in color cast images. An objective measure called color cast could be independent of the illumination condition. There are different assumptions present in the literature to provide an estimate of the Red cast, Blue cast and Green cast present in the image. These assumptions sometimes failed to eliminate the effect of color cast under varying illumination condition. The proposed method performs well under these conditions which may involve two steps. Firstly it performs the color cast detection and thereafter, a power law transformation is introduced to achieve the true color distribution of the image. In our approach a new transformation method which corrects the color cast as well as improves the quality of image. The uncertainty in the color distribution due to the illumination is removed on the basis of fuzzy Gaussian membership functions and further, improves by using weighing distribution. The performance analysis of the proposed approach is carried out by using two objective measures the image distance metric criteria of CIELAB color space and the image quality assessment function. The effectiveness of the proposed approach is illustrated by minimizing the image distance between the reference image and corrected image. The computed higher value of the image quality assessment function obtained in the proposed algorithm application on images, also justifies that the proposed algorithm results the better quality of the image in comparison to existing methods.
\end{abstract}

Keywords: Color, Fuzzy Logic, Correction

\section{Introduction}

The color cast quality metric of images is an important feature in the application area like digital camera variants, biomedical and other practical problems field. The main cause of change in color information of the image is the variation in source illumination. The atmospheric conditions are random in nature. Thus, an image captured with camera at variant weather conditions like underwater images results the deviation in the color quality, visibility, contrast and brightness. The human visual system (HVS) have inherent ability to adjust the said deviation to a certain limit to minimize the impact of different light conditions on the color and hence can perceived the true color. The image acquisition devices at the front end of monitoring system, however, do not have this automatic adjustment ability. Thus, it is an essential objective to perform color correction in order to reproduce the same color image formation in the presence of different light characteristics.

There are some automated tools are also present to correct the color cast like adobe Photoshop, Picasa etc. But the automation doesn't work as desired. It is difficult to control the effect of color cast due to the illumination conditions. Many researchers worked on color cast problem [1-6]. The existing techniques used for color correction are max-RGB [3, 4], Grey World assumption [5], Grey Edge [6], Shades of Grey [7] etc. It may not provide a better solution in terms of removing a particular cast like red, green or

\footnotetext{
E-mail address: sharmaisnitin@gmail.com

ISSN: $1791-2377$ @ 2017 Eastern Macedonia and Thrace Institute of Technology. All rights reserved. doi:10.25103/jestr.103.16
}

blue. Although these techniques can be effective to solve the problem of color cast. Single parameter cannot control the color correction at different level. These techniques may be extended by using the combination with different weights [8]. The weights are applied with the combination of different algorithm. The computation of weights is complex and computationally expensive. These weights are dependent on the classification of images. This classification involved high dimension problem. Jonathan et al. [9] proposed the selection of white patch, the gray world and the max RGB color correction algorithms on dark images in accordance with the actual content of an image but not much effective for the color cast images. Since all algorithm developed have certain assumptions and hence, no algorithm is to be called as a universally true algorithm. In the weighting approach, finding suitable weights for combination is a difficult and error prone task. In other word, it is important to combine the algorithms that have good performance on the input image.

More recently, for color image enhancement [11], O. P. Verma et al. [12] proposed to learn the parameters of membership function used for fuzzification and Rajni et al. [13] proposed the color correction for underwater images. Since the systems based on fuzzy logic are resembled with human decision. In this work, we have proposed a fuzzy based color cast correction algorithm in RGB model that perform an efficient color cast detection and correction in compare to the state of art methods. Firstly, the color cast detection using fuzzy inference system (FIS) is evaluated and thereafter, a new power law transformation is introduced to achieve true color distribution of the image. The uncertainty in the color distribution due to the illumination is removed on the basis of fuzzy Gaussian membership functions and further, improves by using 
weighing distribution. The color distributions are balanced by defining the fuzzy rule based system. By controlling the weights depending on regional properties, we investigate that a visually better result is obtained. The basic idea is to give larger weights to the pixels in noticeable regions where the color cast is high, so that well captured regions are kept intact and others are changed according to the manipulated regions.

The paper is as organized as follow. The existing algorithms for color cast correction are described in Section 2. Section 3 discusses the fuzzy logic and fuzzy rules. Later in Section 4, the proposed algorithm is applied for color correction together with state of the art comparisons and provides significant results. Finally, conclusions drawn from the results obtained are mentioned in Section 5 .

\section{Color Cast Correction Algorithm}

In this section, we discussed the estimation of illumination of different color constancy methods are discussed. The limitation of gray world based approach merits and limitations are also discussed.

\subsection{Estimation of illumination based algorithms}

Image is formed by multiplication of illumination component and reflectance component. To estimate the illuminated component which depends on the illuminant spectral power distribution $\mathrm{P}(\lambda)$ and camera sensitivity $c(\lambda)$. The image $I_{k}(x, y)$ is given by

$$
I_{k}(x, y)=\int_{\text {vidib nages }} P(\lambda) c(\lambda) d \lambda
$$

For Lambertian surface, the image ${ }_{I}(x, y)=(R, G, B)$ are dependent on the light source $e(\lambda)$, the surface reflectance $s(x, y, \lambda)$ and the camera sensitivity functions $\mathrm{c}(\lambda)$ where $\lambda$ is the wavelength. Thus, the image $I_{k}(x, y)$ is given by,

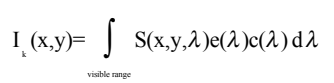

As the integral term involves two unknown terms $\mathrm{e}(\lambda)$ and $\mathrm{S}(\mathrm{x}$, $\mathrm{y}, \lambda)$, thus, integral becomes an under constrained problem, that means integral cannot be solved without any further assumptions. Many color constancy methods have been proposed to estimate the illuminant. The grey world algorithm is just based on the grey world assumption, i.e. the average reflectance in a scene is achromatic. Finlayson et al. [16] proposed a more general color constancy algorithm called Shades of grey (SoG), which can include grey world and Max RGB based on the Minkowski-norm. The general estimation approach can be expressed as

$$
\left(\int\left|\frac{\partial^{n} I^{\sigma}(x)}{\partial x^{n}}\right|^{p} d x\right)^{\frac{1}{p}}=A e^{n, p, \sigma}
$$

Where, $n$ is the order of spatial derivative, $p$ is the Minkowski norm which measures the relative weights and $\sigma$ is the parameter for smoothing the image. When $\mathrm{n}$ is zero, $\sigma$ is zero and $\mathrm{p}=1$ then the expression (3) represents the gray world assumptions, otherwise if it has nonzero finite value then expression represents the shades of gray. If $p$ becomes infinite then expression is of a max-RGB.

\subsection{Gray World Based Assumption Algorithm}

The assumption made in the gray world algorithm is that the given color image has a sufficient amount of color variations. In the present application we have considered Red (R) Green (G) and Blue (B) color model of color space. The mean value of each $R, G$, and $B$ component of the image should have a common mean intensity of the image. In a real world, this assumption for images may hold true. Color balancing algorithms can also create images to have a uniform gray value for its $\mathrm{R}, \mathrm{G}$, and B channel. If the image is captured by a digital camera under a particular lighting condition, the effect of the special lighting cast can be removed by taking the mean value of each channel. As a result, it estimates the color of the image linearly which is much closer to the original scene. In terms of implementation, the mean value $\bar{R}, \bar{G}$ and $\bar{B}$ of red, green and blue channel respectively, of a color image of size $\mathrm{M} x$ $\mathrm{N}$, can be calculated as

$$
\begin{aligned}
& \bar{R}=\frac{1}{M N} \sum_{i=1}^{M} \sum_{j=1}^{N} R(i, j) \\
& \bar{G}=\frac{1}{M N} \sum_{i=1}^{M} \sum_{j=1}^{N} G(i, j) \\
& \bar{B}=\frac{1}{M N} \sum_{i=1}^{M} \sum_{j=1}^{N} B(i, j)
\end{aligned}
$$

Where, $i$ and $j$ denotes the indices of pixel position. The cast arises here is due to non-linear behaviour of the different illumination conditions. This problem cannot completely solve with the gray world assumption.

The mean intensity of an image is given by

$$
\overline{\mathrm{W}}=(\mathrm{R}+\mathrm{G}+\mathrm{B}) / 3
$$

\section{Proposed Algorithm}

At the time of image acquisition, each camera uses its own RGB space that is converted to a standard RGB space later after achieving color constancy, this shows that the transformation between $\mathrm{R} / \mathrm{G} / \mathrm{B}$ spaces, which requires calibrating the imaging device. Therefore, it will inevitably fail on those images from any unknown camera. The proposed approach first detects the presence of dominance of color in the given image without any preassumptions and then corrects the color cast in the image in the same space. We first detect the color cast of the image depending on the color dominance presence. Most of the algorithms used for finding the cast are depends on constant value as given in equation (3).The constant value based on the assumption. The effect of color cast is uncertain which is perceived by the human eye. The vagueness in perception is increased under varying illumination condition. Here, we reduced the vagueness of perception by using Fuzzy rules. These rules calculate the color cast on the basis of intensity value. Thereafter, remove the color cast in such a way that the true distribution of colors present in the image does not get altered. Further, we applied a correction factor which behaves nonlinearly for obtained the color corrected enhanced image. The correction factors have been formed with the combination of base and the weighting distribution. The weighting distribution is introduced to reduce the effect of color cast. Finally, the color corrected enhanced image is obtained. 


\subsection{Color Cast Detection}

In order to detect the dominant color, first we need to calculate the normalized value of $\mathrm{R}, \mathrm{G}$, and $\mathrm{B}$ channel from the given input color cast image henceforth referred to as "test image". Then the mean intensity of each color channel $\overline{\mathrm{R}}, \overline{\mathrm{G}}$ and $\overline{\mathrm{B}}$ is calculated. In this work, the fuzzy approach is included for the decision of dominance of color presence. Each color channel is fuzzified by using Gaussian membership function. We use Gaussian membership function as shown in Fig.1 to avoid boundary value and represent vague or linguistic terms. Gaussian membership function is given by:

$$
\mu_{\mathrm{A}}(\mathrm{x})=\exp \left(\frac{(\mathrm{c}-\mathrm{x})^{2}}{2 \sigma^{2}}\right)
$$

Here, $\mathrm{c}$ and $\sigma$ are centre and width of the fuzzy set $\mathrm{A}$ and $\mathrm{x} \in \mathrm{A}$

The maximum number of pixels belongs to either higher or lower side of membership function, the Gaussian fuzzy sets where, $\mathrm{x} \in\{0,1\}$ and standard deviation oare 0.19667 . The fuzzy rules of the proposed Mamdani fuzzy inference system are defined in Table 1.

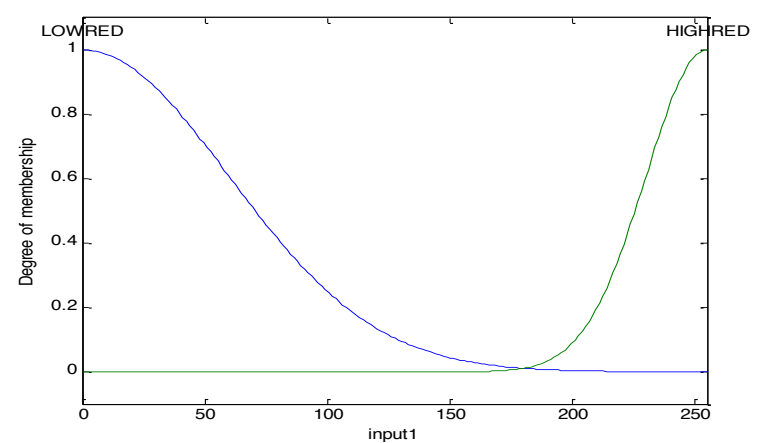

Fig. 1. Gaussian membership function curve for the LOW and the HIGH intensity values

Table 1. Color cast determination fuzzy rules

\begin{tabular}{|c|c|c|c|c|}
\hline S.No. & $R(i, j)$ & $G(i, j)$ & $B(i, j)$ & Color cast \\
\hline 1 & HIGH & LOW & LOW & Red \\
\hline 2 & LOW & $\mathrm{HIGH}$ & LOW & Green \\
\hline 3 & LOW & LOW & $\mathrm{HIGH}$ & Blue \\
\hline 4 & $\mathrm{HIGH}$ & HIGH & LOW & Red and Green \\
\hline 5 & LOW & HIGH & HIGH & $\begin{array}{l}\text { Green and } \\
\text { Blue }\end{array}$ \\
\hline 6 & HIGH & LOW & $\mathrm{HIGH}$ & Red and Blue \\
\hline
\end{tabular}

Generally, the intensity levels are not well spread with the correction of color cast. The pixel intensity as shown in table1 defines the belonging of the cast. The cast of each pixel evaluates by using membership function. The higher the pixel intensity greater the membership value and vice versa. After evaluation of color cast, we find the mean value of each channel as defined in equation (4), equation (5) and equation (6). Now depending on the region, a proposed base value is defined in Table 2 .

Table 2. Proposed base value for the different region of casts

\begin{tabular}{|l|l|l|}
\hline S.No. & Region & Base \\
\hline 1 & Region 1 & $\bar{R}$ \\
\hline 2 & Region 2 & $\bar{G}$ \\
\hline 3 & Region 3 & $\bar{B}$ \\
\hline 4 & Region 4 & $\bar{R}+\bar{G}$ \\
\hline
\end{tabular}

\begin{tabular}{|l|l|l|}
\hline 5 & Region 5 & $\frac{\bar{G}+\bar{B}}{2}$ \\
\hline 6 & Region 6 & $\frac{\bar{R}+\bar{B}}{2}$ \\
\hline
\end{tabular}

Accordingly, the color cast of the image is obtained as an output after the defuzzification and aggregation process using the above defined fuzzy rules. The output membership function is shown in Fig. 2. The rules depend on the degree of membership function. The overall cast evaluates the rule under membership function. The higher number of pixels decides the cast of the image.

Color Cast Correction- After the finalization of cast type, color cast of image is corrected by applying correction factor $\alpha_{\mathrm{r}}, \alpha_{\mathrm{g}}$ and $\alpha_{\mathrm{b}}$ on red, green and blue channel respectively are obtained from equation given by,

$$
\alpha_{\mathrm{r}}=\frac{\overline{\mathrm{R}}}{\text { base }}, \alpha_{\mathrm{g}}=\frac{\overline{\mathrm{G}}}{\text { base }} \text { and } \alpha_{\mathrm{b}}=\frac{\overline{\mathrm{B}}}{\text { base }}
$$

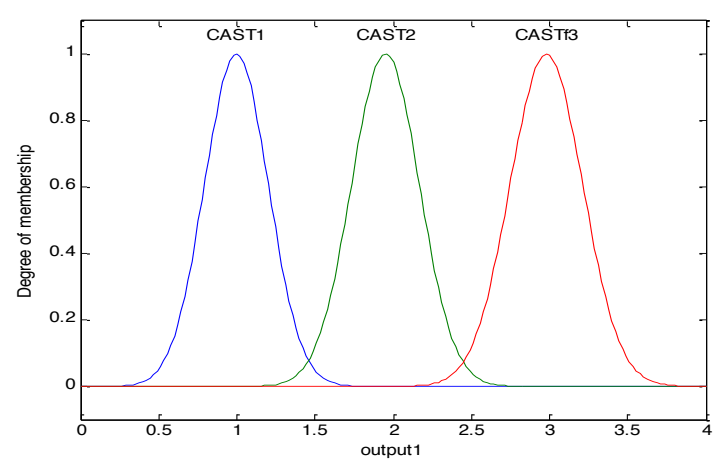

Fig. 2. Gaussian membership function for output color cast- Red cast, Green cast and Blue cast

These factors are depending on the base of the channel. This base has the value of color cast obtained in the first operation of proposed approach that is the color cast detection. This color cast effect is corrected by using the equation (10).

$$
\begin{aligned}
& \left.\tilde{R}(i, j)=R(i, j){ }^{* / w_{r}}, \tilde{G}(i, j)=G(i, j)\right)^{\star_{s} / w_{s}} \\
& \text { and } \tilde{B}(i, j)=B(i, j))^{\star_{b} / w_{b}}
\end{aligned}
$$

Where, $\mathrm{w}_{\mathrm{r}}, \mathrm{w}_{\mathrm{g}}$, and $\mathrm{w}_{\mathrm{b}}$ are the color correction factor that reduced the effect of color cast. The power law coefficient is determined by the dominant color detected by fuzzy color correction factor to the weighted factor given in equation (11). Whereas, correction factor is obtained by computing the ratio of the mean value of intensity of color channels present on base depend in the actual image. The value of $\mathrm{w}_{1}, \mathrm{w}_{2}$, and $\mathrm{w}_{3}$ is chosen as per the type of cast. If the resulted image is Red cast then $\mathrm{w}_{\mathrm{r}}=\overline{\mathrm{W}}$ and $\mathrm{w}_{\mathrm{g}}=1$ and $\mathrm{w}_{\mathrm{b}}=1$, image is Green cast then $\mathrm{w}_{\mathrm{r}}=1, \mathrm{w}_{\mathrm{g}}=\overline{\mathrm{W}}$ and $\mathrm{w}_{\mathrm{b}}=1$. Similarly in case of blue cast $\mathrm{w}_{\mathrm{r}}=1, \mathrm{w}_{\mathrm{g}}=1$ and $\mathrm{w}_{\mathrm{b}}=\overline{\mathrm{W}}$. This weighing factor $\overline{\mathrm{W}}$ is determined from equation (7). This factor gives mean intensity of the test image. Mathematically the weighing factor is calculated as

$$
\text { if } \alpha_{i}=1 \text {, then } w_{i}=\bar{W} \text { and } w_{j}=1, j \neq i \text {. where } i, j \in\{r, g, b\}
$$

Finally, the corrected pixel values $\tilde{R}(i, j), \tilde{G}(i, j)$ and $\tilde{B}(i, j)$ of each color channel is obtained. This law maintains the correction 
with wide intensity range. We calculate the correction factor as in the gray world based correction but apply directly on each intensity value non- linearly. Hence the color cast corrected image is obtained.

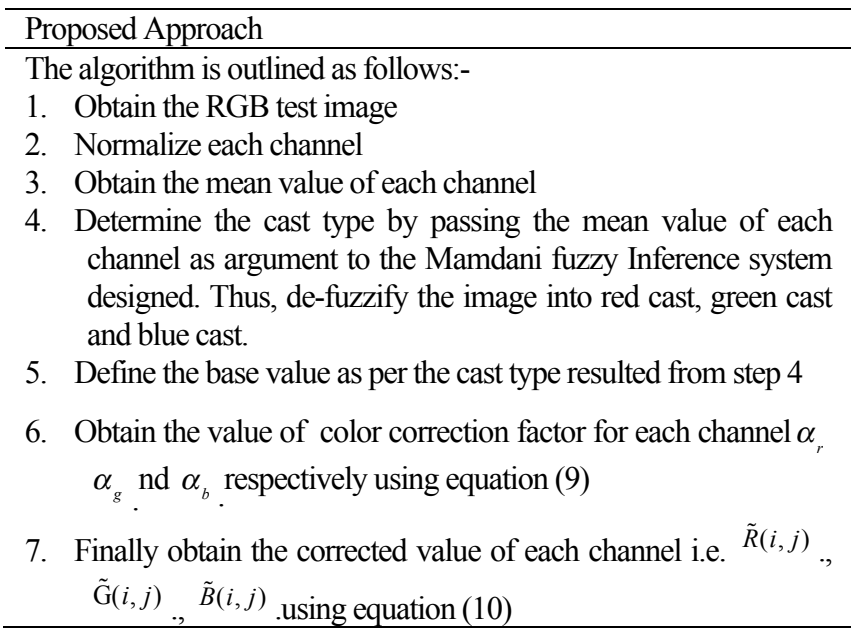

\section{Performance Measures:-}

The two image quality performance measures are considered to evaluate the effectiveness of the proposed approach. These performance measures are comprehensive image quality assessment model and CIELAB metric.

Comprehensive Image Quality Assessment Model:-The color quality of the image is measured subjectively as well as objectively. The subjective evaluation is based on the human perception of the image. It may vary from individual to individuals. The objective evaluation is more reliable, consistent and replicable especially when the goal is to compare with the other existing approaches. The comprehensive image quality parameters are proposed in the literature [14].A comprehensive image quality assessment model, called as the best quality assessment function for the color image was established. The quality parameters like luminance, contrast and entropy are quality evaluator and are described below Average Luminance

The average luminance of an image $\overline{\mathrm{L}}$ is given by:-

$\bar{L}=\frac{1}{M N} \sum_{i=0}^{M-1} \sum_{j=0}^{N-1} L(i, j)$

Where, $\mathrm{M}$ and $\mathrm{N}$ are the number of rows and columns of an image respectively.

\section{Average Information Entropy}

In color images, information entropy evaluates for each channel red, green and blue is given by:-

$$
H=-\sum_{i=0}^{255} p(i) \log _{2} p(i)
$$

where, $p(i)$ is the probability distribution of the pixel information for three bit image. Thereafter, take the average information of three channels $(\bar{H})$.

\section{Average Contrast:}

The color contrast can be calculated for each RGB channel are given by,

$$
\vec{C}_{c}(\mathrm{i}, \mathrm{j})=\nabla R(i, j) \hat{\mathrm{x}}+\nabla G(i, j) \hat{y}+\nabla B(i, j) \hat{z}
$$

Where, $\nabla R, \nabla G$ and $\nabla B$ are the gradient of red, green and blue channel respectively. The total contrast computes by taking the average of these channels.

$$
C_{c}=\left\|\bar{C}_{C}\right\|=\sqrt{\frac{\nabla^{2} R+\nabla^{2} G+\nabla^{2} B}{3}}
$$

\begin{tabular}{|c|c|c|c|c|c|c|}
\hline & & & $\begin{array}{l}\text { Average } \\
\text { Luminance } \\
\bar{L}\end{array}$ & $\begin{array}{l}\text { Average } \\
\text { Entropy } \\
\bar{H}\end{array}$ & $\begin{array}{l}\text { Average } \\
\text { Contrast } \\
\bar{C}\end{array}$ & $\mathrm{CAF}$ \\
\hline \multirow[t]{15}{*}{ Two men } & \multirow[t]{5}{*}{ Red Cast } & Test Image & 105.874 & 7.6176 & 117.6348 & 14.3537 \\
\hline & & Proposed Approach & 121.4628 & 7.8592 & 128.8760 & 22.8940 \\
\hline & & Grey world & 114.6916 & 7.4689 & 126.3866 & 18.2284 \\
\hline & & Max-RGB & 105.8476 & 7.5950 & 116.4403 & 14.2747 \\
\hline & & Shades of Grey & 106.1014 & 7.7031 & 123.5935 & 14.8013 \\
\hline & \multirow{5}{*}{$\begin{array}{l}\text { Green } \\
\text { Cast }\end{array}$} & Test Image & 154.3369 & 7.7214 & 107.404 & 12.2340 \\
\hline & & Proposed Approach & 139.3876 & 7.7641 & 113.2544 & 18.8837 \\
\hline & & Grey world & 147.5456 & 7.6409 & 103.9105 & 14.6033 \\
\hline & & Max-RGB & 154.2861 & 7.7219 & 107.5911 & 12.2576 \\
\hline & & Shades of Grey & 150.8933 & 7.7248 & 106.0571 & 13.4952 \\
\hline & \multirow[t]{5}{*}{ Blue Cast } & Test Image & 95.2479 & 7.6093 & 124.4972 & 10.5967 \\
\hline & & Proposed Approach & 122.7724 & 7.8828 & 127.7406 & 23.6610 \\
\hline & & Grey world & 116.2059 & 7.4491 & 137.1300 & 19.2994 \\
\hline & & Max-RGB & 95.3044 & 7.5772 & 121.0267 & 10.4963 \\
\hline & & Shades of Grey & 101.4161 & 7.6467 & 130.5941 & 13.0091 \\
\hline \multirow{3}{*}{$\begin{array}{l}\text { Color } \\
\text { checker }\end{array}$} & \multirow[t]{3}{*}{ Red Cast } & Test Image & 117.0651 & 6.9913 & 44.2898 & 18.0358 \\
\hline & & Proposed Approach & 136.8766 & 7.3210 & 51.4229 & 19.6046 \\
\hline & & Grey world & 126.2622 & 7.1793 & 53.9270 & 19.4550 \\
\hline
\end{tabular}

Table 3. Comparison of luminance, entropy, average contrast and CAF value for two different images using proposed, grey world shades of gray and Max-RGB methods 
Om Prakash Verma and Nitin Sharma/Journal of Engineering Science and Technology Review 10 (3) (2017) 115-122

\begin{tabular}{|c|c|c|c|c|c|}
\hline & Max-RGB & 116.9710 & 7.0403 & 46.3699 & 18.3716 \\
\hline & Shades of Grey & 117.4282 & 7.1331 & 49.4301 & 18.9138 \\
\hline \multirow{5}{*}{$\begin{array}{l}\text { Green } \\
\text { Cast }\end{array}$} & Test Image & 139.2496 & 7.0017 & 42.9251 & 17.92191 \\
\hline & Proposed Approach & 132.1887 & 7.3055 & 49.7295 & 19.4002 \\
\hline & Grey world & 124.4725 & 7.1858 & 48.8869 & 19.0008 \\
\hline & Max-RGB & 136.9524 & 7.0310 & 43.6200 & 18.0696 \\
\hline & Shades of Grey & 125.7044 & 7.1179 & 45.1199 & 18.4477 \\
\hline \multirow[t]{5}{*}{ Blue Cast } & Test Image & 101.8550 & 7.1648 & 47.7581 & 18.8349 \\
\hline & Proposed Approach & 132.1369 & 7.3283 & 51.2518 & 19.6078 \\
\hline & Grey world & 130.3048 & 6.1334 & 45.3594 & 15.9172 \\
\hline & Max-RGB & 125.5105 & 6.1117 & 36.1218 & 14.9832 \\
\hline & Shades of Grey & 122.2703 & 6.1470 & 39.4600 & 15.4065 \\
\hline
\end{tabular}

Table 4. Comparison of $\triangle E$ CIELAB metric for Proposed Approach, grey world, max-RGB and shades of grey

\begin{tabular}{|c|c|c|c|c|c|c|}
\hline & & $\begin{array}{l}\Delta E_{r c} \\
\text { (test) }\end{array}$ & $\begin{array}{l}\Delta E_{r e} \\
\text { (proposed Approach) }\end{array}$ & $\begin{array}{l}\Delta E_{r g} \\
\text { (grey) }\end{array}$ & $\begin{array}{l}\Delta E_{r m} \\
\text { (max-RGB) }\end{array}$ & $\begin{array}{l}\Delta E_{r c} \\
\text { (shades of grey) }\end{array}$ \\
\hline \multirow[t]{3}{*}{ Two men Image } & Red Cast & 0.1147 & 0.0738 & 0.0922 & 0.1146 & 0.0774 \\
\hline & $\begin{array}{l}\text { Green } \\
\text { Cast }\end{array}$ & 0.0968 & 0.0577 & 0.0854 & 0.0983 & 0.0896 \\
\hline & Blue Cast & 0.2241 & 0.0479 & 0.1329 & 0.2227 & 0.1607 \\
\hline \multirow{3}{*}{$\begin{array}{l}\text { Color } \\
\text { Image }\end{array}$} & Red Cast & 0.1996 & 0.0637 & 0.1041 & 0.1992 & 0.1318 \\
\hline & $\begin{array}{l}\text { Green } \\
\text { Cast }\end{array}$ & 0.1414 & 0.0602 & 0.0724 & 0.1412 & 0.1030 \\
\hline & Blue Cast & 0.2571 & 0.1019 & 0.1425 & 0.2572 & 0.1308 \\
\hline
\end{tabular}

The average color contrast for each channel of an RGB image are given by

$$
\bar{C}=\frac{1}{M N} \sum_{i=0}^{M-1} \sum_{j=0}^{N-1} C_{C}(i, j)
$$

Where, $\bar{C}$ represents average contrast of the image. The color image quality assessment function defined in [14].

$$
C A F=\bar{H}^{\alpha}+\bar{C}^{\beta}+\bar{N}
$$

The higher CAF value gives better image quality. This value is computed by adding the parameters like luminance, entropy, contrast and noise.

CIELAB Metric: -This metric estimates accuracy of the color reproduction in comparison to the original when analysed by a human observer [15]. This metric is suitable for measuring color difference of large uniform color targets. CIELAB is based on one channel for Luminance (L) and two color channels (a and b). The $a$-axis starts from green (-a) to red (+a) and the b-axis starts from blue (-b) to yellow $(+b)$. The Luminance $(\mathrm{L})$ starts from the bottom to the top of the three-dimensional model. The $\Delta E_{c}$ and $\Delta E_{e}$ metric are given by
$\Delta E_{c}=\sqrt{\Delta L_{c}^{2}+\Delta a_{c}^{2}+\Delta b_{c}^{2}}$

(17)

where,

$$
\begin{aligned}
& \Delta L_{c}=L_{\text {cast }}-L_{\text {original }} \\
& \Delta a_{c}=a_{\text {cast }}-a_{\text {original }} \\
& \Delta b_{c}=b_{\text {cast }}-b_{\text {original }} \\
& \Delta E_{e}=\sqrt{\Delta L_{e}^{2}+\Delta a_{e}^{2}+\Delta b_{e}^{2}}
\end{aligned}
$$

where,

$$
\begin{aligned}
& \Delta \mathrm{L}_{\mathrm{e}}=\mathrm{L}_{\text {enhanced }}-\mathrm{L}_{\text {orignal }} \\
& \Delta \mathrm{a}_{\mathrm{e}}=\mathrm{a}_{\text {enhanced }}-\mathrm{a}_{\text {orignal }} \\
& \Delta \mathrm{b}_{\mathrm{e}}=\mathrm{b}_{\text {enhanced }}-\mathrm{b}_{\text {original }}
\end{aligned}
$$

Parameters $\Delta \mathrm{L}_{\mathrm{c}}, \Delta \mathrm{a}_{\mathrm{c}}$ and $\Delta \mathrm{b}_{\mathrm{c}}$ is the difference in the cast and test image coordinates of $\mathrm{L}, \mathrm{a}$, and $\mathrm{b}$ of CIELAB color space and $\Delta \mathrm{L}_{\mathrm{e}}, \Delta \mathrm{a}_{\mathrm{e}}$ and $\Delta \mathrm{b}_{\mathrm{e}}$ are the coordinates $\mathrm{L}$, The ' $\mathrm{a}$ ' and ' $\mathrm{b}$ ' of enhanced image in CIELAB color space. $\Delta E$ is the Euclidean distance for measuring the difference between colours. Smaller value of $\Delta \mathrm{E}$ indicates that the enhanced test image is closer to the reference image.

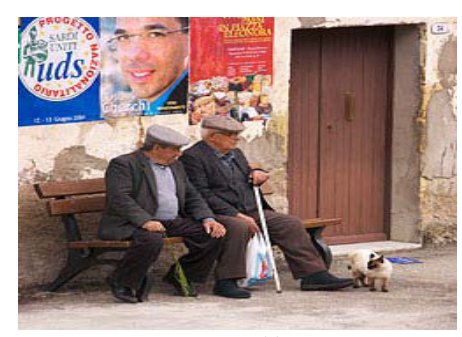

(a) 

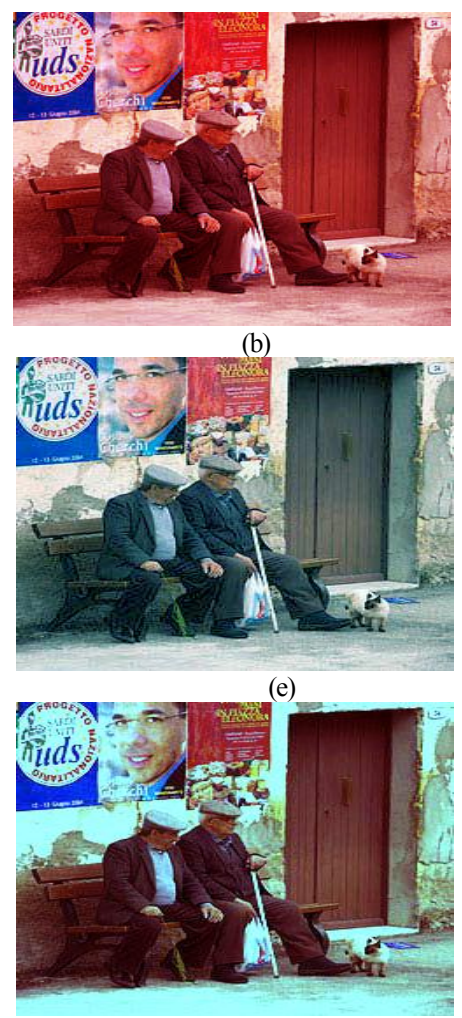

(h)

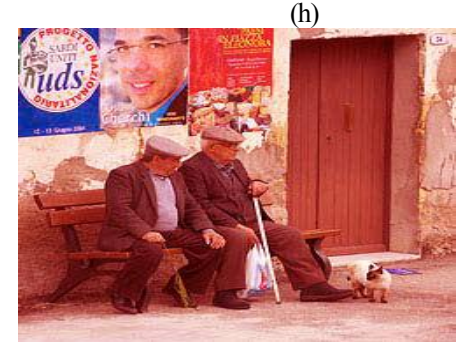

(k)

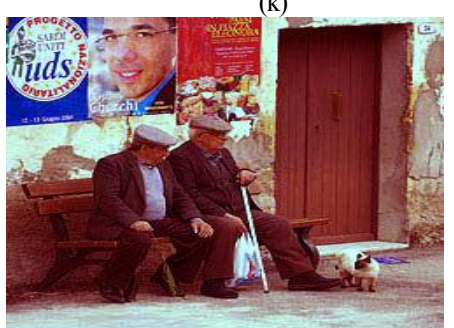

(n)

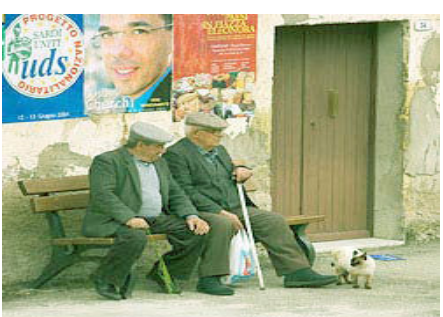

(c)
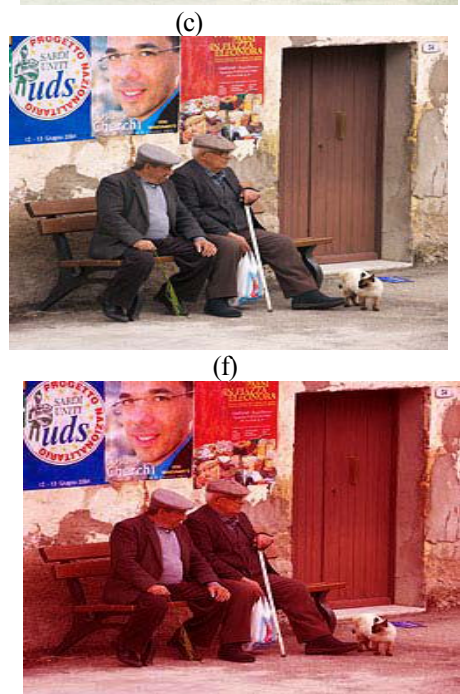

(i)

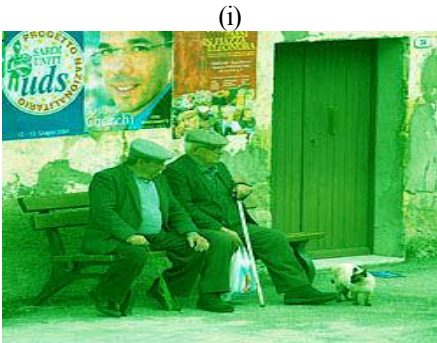

(1)

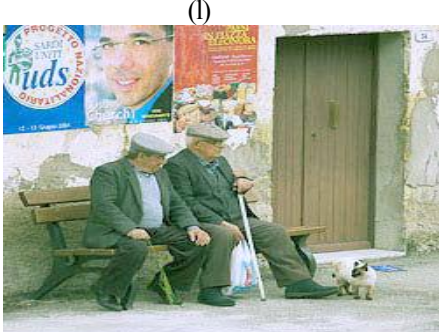

(o)
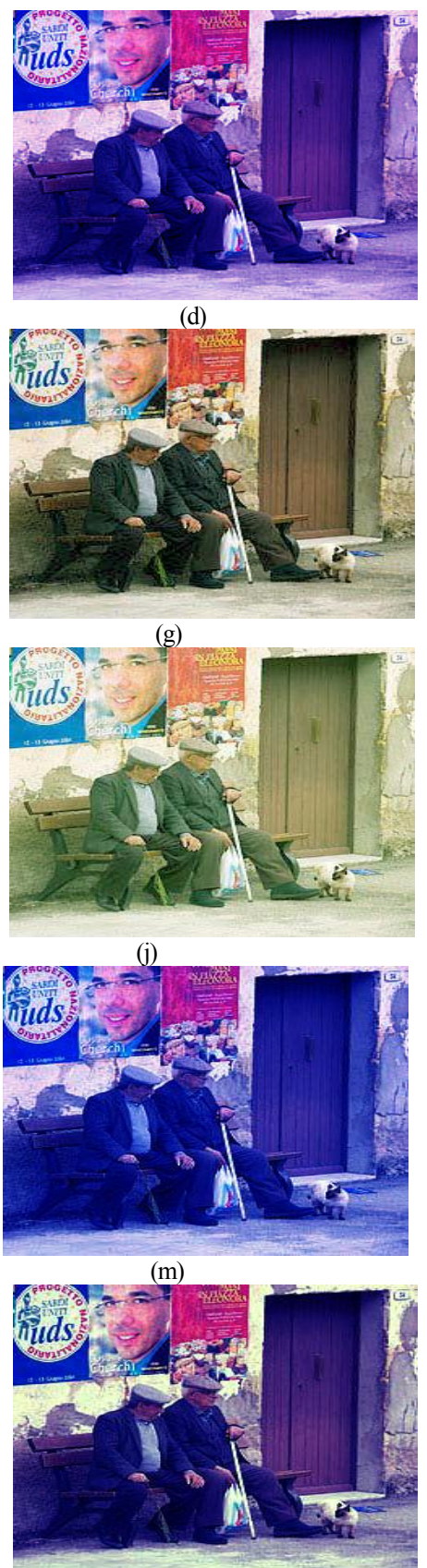

(p)

Fig. 3. Two-men image (a) Reference, (b) Red cast (c) Green cast, (d) Blue cast, (e) proposed corrected Red cast (f) proposed corrected Green cast (g) proposed corrected Blue cast (h) Grey world corrected Red cast (i) Grey world corrected Green cast (j) Grey world corrected Blue cast (k) max-RGB corrected Red cast (l) max-RGB corrected Green cast Two-men image (m) max-RGB corrected Blue cast, (n) shades of grey corrected Red cast, (o) shades of grey approach corrected Green cast (p) shades of grey approach corrected Blue cast

\section{Results and Discussions}

In this section experimental results are presented to prove the effectiveness of the proposed color correction algorithm on the natural images under red, green and blue cast. The proposed approach has been successfully implemented on Intel core i3 at $2.40 \mathrm{GHz}$ using MATLAB version 2014. All images used in experiment are cast using paintshop pro software and evaluate the luminance, entropy, average contrast and CAF values. The color corrected images are shown in Fig. 3 and Fig. 4.The reference image is the original image without any bad illumination effect. The distorted images are obtained by adding color cast to them. The distorted images are corrected by some well-known methods in literature for color correction: max-RGB, Grey World assumption, Grey Edge, Shades of Gray and the proposed corrected approach. The image quality parameters: luminance, entropy, average contrast and CAF value for two different images are summarized in Table-3. The results show a good match with human ground truth with proposed technique. The higher entropy value is obtained as compared with existing methods. An additional performance measure $\Delta E$ had been taken in which image distances are calculated using equation (17), equation (18) and are summarized in Table-4. It is observed that the distance is significantly reduced with the proposed approach. The reference two men image is shown in Fig. 3(a). The red, green and blue cast images are shown in Fig.3 (b), (c) and (d) respectively having entropy of 7.6176, 7.7214 and 7.6093 respectively, are efficiently corrected with proposed technique. The images shown in Fig.3 (e), (f) and (g) are the resulted color cast corrected images using proposed technique.

It is clearly observed that proposed technique removes the color cast correctly and it is confirmed from the metric results 
obtained that is the entropy value of the corrected images obtained are 7.8592, 7.7614 and 7.8828 respectively. The overall contrast values of the test images are 14.3537, 12.2340 and 10.5967.And these values are significantly enhanced in the corrected images to $22.8940,18.8837$ and 23.6610 respectively. The color of the over coat in two men image under different cast are appear slightly black but after applying the proposed method the color of over coat becomes truly black as it appears in the reference image. The same result can be inferred by employing the proposed technique over the color Color checker image. Images resulted after processing through the proposed technique are shown in Fig. 4 (e), (f) and (g),

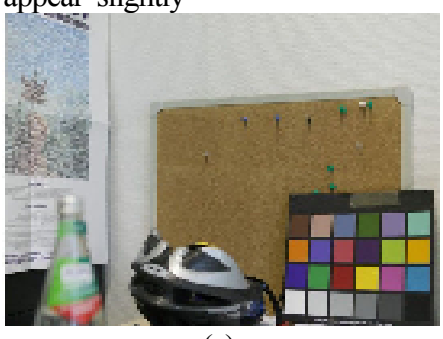

(a)

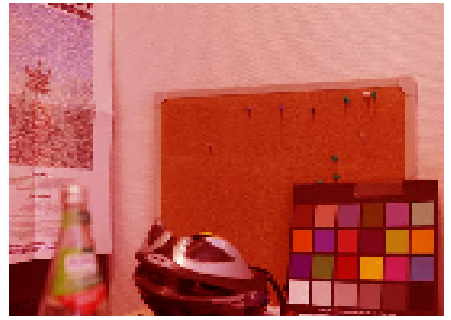

(b)

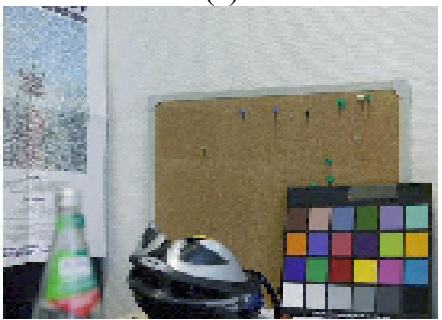

(e)

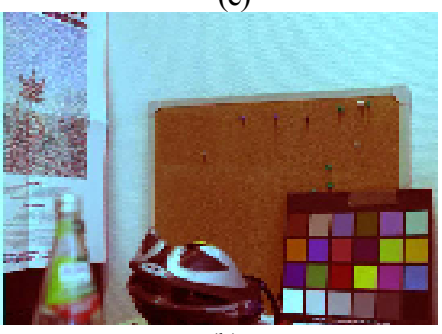

(h)

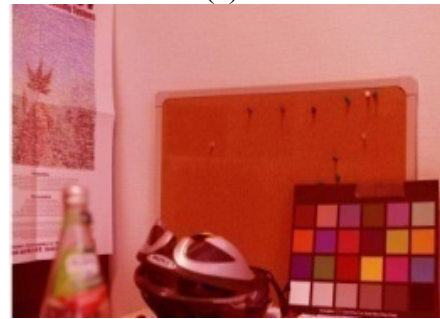

(k)

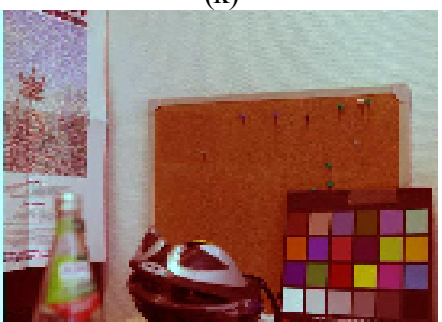

(n)

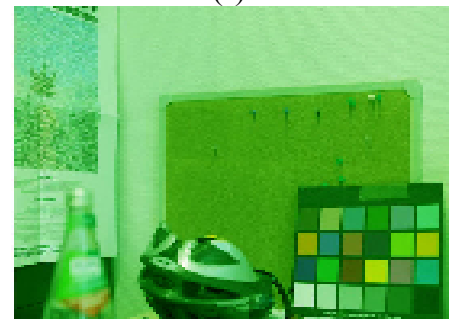

(c)

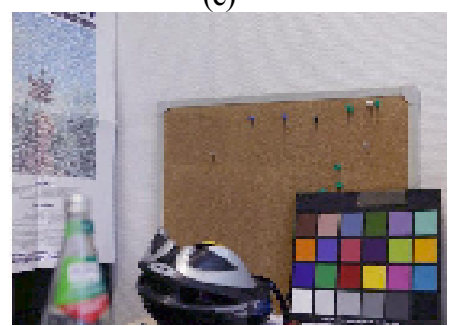

(f)

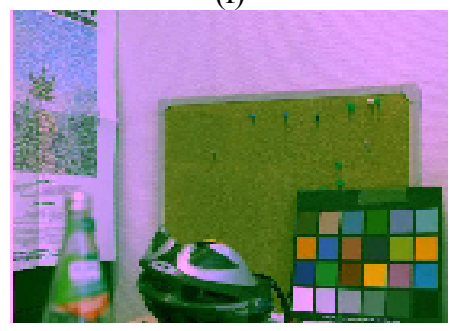

(i)

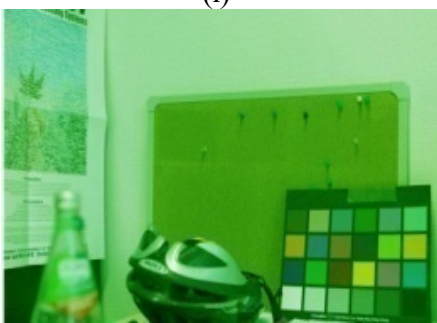

(l)

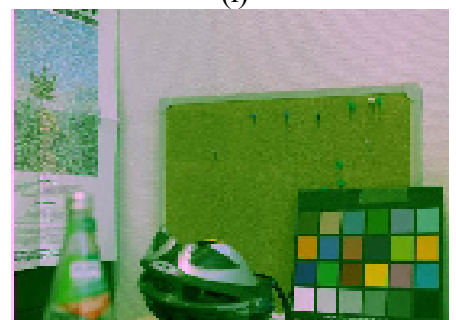

(o)

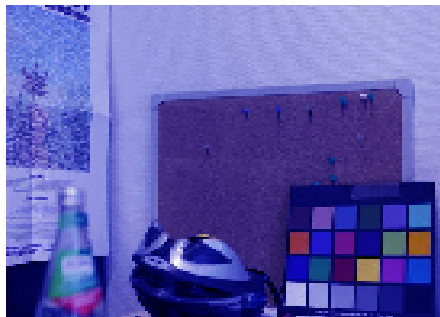

(d)

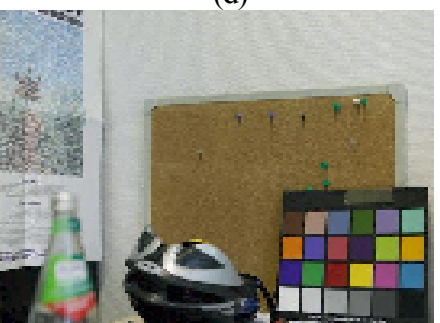

(g)

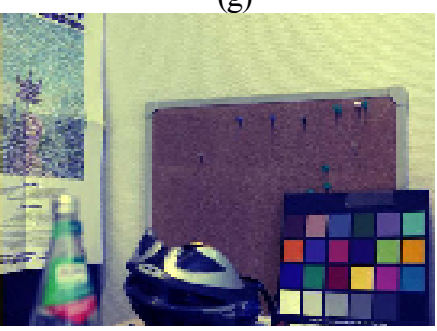

(j)

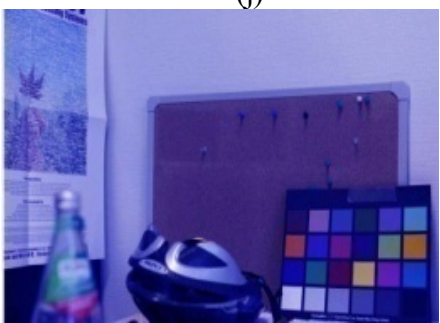

(m)

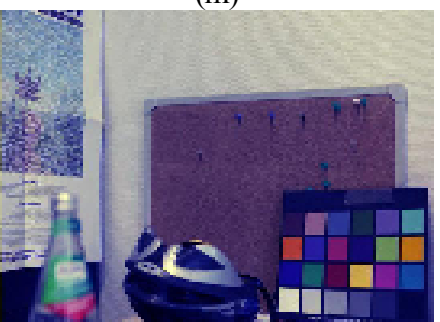

(p)

Fig.4-Color checker image (a) Reference, (b) Red cast, (c) Green cast, (d) Blue cast, (e) proposed corrected Red cast (f) proposed corrected Green cast (g) proposed corrected Blue cast (h) Gray corrected Red cast (i) Gray corrected Green cast (j) Gray corrected Blue cast (k) max-RGB corrected Red cast (l) max-RGB corrected Green cast (m) max-RGB corrected Blue cast, (n) shades of grey corrected Red cast, (o) shades of grey approach corrected Green cast (p) shades of grey approach corrected Blue cast 
It is clearly visible that proposed technique effectively remove the color cast and retain the originality of the image. Statistically obtained color corrective measures like entropy, contrast for corrected Color checker images are 7.3210, 7.3055, 7.3283 and 19.6046, 19.4008, 19.6078 respectively. The obtained CAF values of proposed algorithm are highest as compared to all other existing methods for all images.

\section{Conclusions}

In this paper, the Fuzzy based approach is used for detecting and removing all types of color cast. This algorithm detects the actual cast on the image in an effective manner without assuming it like Reddish, Greenish and Bluish. The uncertainty in the cast further removed completely by using the fuzzy logic. Thus, we recover true color distribution of the image and develop algorithm which is robust under different lighting conditions. As a result, the image obtained with high entropy, luminance and contrast. In addition to that the image obtained with high intensity value. The effectiveness of the proposed algorithm is shown by measuring the quality assessment function and CIELAB metric. These performance measures are compared with the existing color cast algorithm. The maximum value of the function is achieved by using proposed approach which shows a better quality of the image. It was inferred from the decrement nature of distance measured through CIELAB metric that the resulted image approaches towards the reference image.

This is an Open Access article distributed under the terms of the Creative Commons Attribution Licence

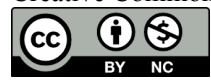

\section{References}

1. Land, E.H., McCann, J.J., Lightness and retinex theory. J. Opt. Soc. Amer. 1974, 61 (1), 1-11.

2. Maloney, L.T., Wandell, B.A. Color constancy: a method for recovering surface spectral reflectance. J. Opt. Soc.Amer.A3., 1986, 3 (1), 29-33.

3. E. H. Land, "The retinex theory of color vision," Scientific American, vol. 237, no. 6, pp. 108-128, December 1977. 1,2

4. B. Funt and L. Shi, "The rehabilitation of maxRGB," in Color and Imaging Conf., 2010, no. 1, pp. 256-259, Soc. Imag. Sci. Technology.

5. G. Buchsbaum, "A spatial processor model for object color perception," Journal of the Franklin Institute, vol. 310, no. 1, pp. 1-26, July 1980. 1, 2

6. G. D. Finlayson and E. Trezzi, "Shades of gray and color constancy," in Proc. IS\&T/SID Color Imaging conference, 2004, pp. 37-41.

7. J. van de Weijer, T. Gevers, and A. Gijsenij, "Edge based color constancy," IEEE Transactions on Image Processing, vol. 16, no. 9, pp. 2207-2214, 2007.

8. A. Gijsenij and T. Gevers Color Constancy Using Natural Statistics and Scene Semantics IEEE Transactions on Pattern Analysis and Machine Intelligence, Vol. 33, No. 4, 2011

9. J. Negrete, R. E. Sanchez-Yanez, "Automatic selection of color constancy algorithms for dark image enhancement by fuzzy rule-based reasoning" Applied Soft Computing, Volume 28, March 2015, pp. 1-10.
10. L. A. Zadeh,"Fuzzy Sets, Information and Control”, 1965, pp. 338-353.

11. Rizzi. A, Gatta. C, Marini. D, "A new algorithm for unsupervised global and local color correction", Pattern Recognition Letters, Vol 24, No. 11, pp.1663-1677, July. 2003.

12. Om Prakash Verma, Puneet Kumar, Madasu Hanmandlu, Sidharth Chhabra, High dynamic range optimal fuzzy color image enhancement using Artificial Ant Colony System, Applied Soft Computing, Volume 12,Issue 1, pp. 394-404 January 2012.

13. Sethi, R., Sreedevi, I., Verma, O. P., \& Jain, V. (2015, December). An optimal underwater image enhancement based on fuzzy gray world algorithm and Bacterial Foraging algorithm, IEEE Fifth National Conference on Computer Vision, Pattern Recognition, Image Processing and Graphics (NCVPRIPG),pp. 1-4 December,2015.

14. Z. X. Xie, Z. F. Wang, "Color Image Quality Assessment Based on Image Quality Parameters Perceived by Human Vision System", International Conference on Multimedia Technology (ICMT), Ningbo 29-31 Oct. 2010.

15. X. M. Zhang, B. A. Wandell, "A spatial extension to CIELAB for digital color image reproduction," Symposium Proceedings, 1996.

16. G.D. Finlayson and E. Trezzi, "Shades of gray and color constancy", Proc. of IS\&T/SID Color Imaging Conference, Scottsdale, Arizona, USA, pp.37-41, 2004. 